Pure and Applied Mathematics Quarterly

Volume 8, Number 3

$693-711,2012$

\title{
Hopf Algebra Structures Over Generalized Path Coalgebras*
}

\author{
Fang Li and Lili Chen
}

\begin{abstract}
The aim of this paper is to characterize the condition under which a generalized path coalgebra holds Hopf algebra structures, see Theorem 2.2. It generalizes the corresponding result on path coalgebra (see [1]). The motivation is to construct a new kind of non-pointed Hopf algebras.

Keywords: Generalized path coalgebra, $Q_{0}$-closed coradical, $G$-type, $\mathcal{C}$ arrow-stable, Hopf algebra.
\end{abstract}

\section{Introduction and Preliminaries}

Generalized path algebras were introduced in [2] and played an important role in describing the structures of artinian (in particular, finite dimensional) algebras [2] [4]. Furthermore, in [5], the dual theory for coalgebras was researched through the notion of generalized path coalgebras. Since the dual of an algebra need not be a coalgebra in general, the theory of generalized path coalgebras cannot be obtained trivially through the dual method from that of generalized path algebras. Path coalgebras are always pointed. One can think the motivation of generalized path coalgebras is to build a new kind of non-pointed Hopf algebras by the main result, Theorem 2.2 , in this paper. It is interesting to the classification problem of Hopf algebras.

Received October 28, 2007.

2000 Mathematics Subject Classifications. 16W30, 16G20.

* Project supported by the National Natural Science Foundation of China (No. 10871170) and the Zhejiang Provincial Natural Science Foundation of China (No. D7080064) 
In this paper, we always assume that $k$ is a field and all linear spaces are over $k$. The symbol $\mathbf{N}$ denotes the set of all non-negative integers.

Let $Q=\left(Q_{0}, Q_{1}\right)$ be a quiver. For each $\beta \in Q_{1}$, let $s(\beta)$ and $t(\beta)$ denote the source and target of the arrow $\beta$ respectively. Let $\mathcal{C}=\left\{C_{i}: v_{i} \in Q_{0}\right\}$ be a family of $k$-coalgebras $C_{i}$ with comultiplication $\Delta_{i}$ and counit $\varepsilon_{i}$, indexed by the vertices of $Q$. The non-zero elements of $\bigcup_{v_{i} \in Q_{0}} C_{i}$ are called the $\mathcal{C}$-paths of length zero. For each $n \geq 1$, a $\mathcal{C}$-path of length $n$ is given by a formal sequence $c_{1} \beta_{1} c_{2} \beta_{2} \cdots c_{n} \beta_{n} c_{n+1}$, where $\beta_{1} \cdots \beta_{n}$ is a path in $Q, 0 \neq c_{i} \in C_{s\left(\beta_{i}\right)}$ for each $i=1, \cdots, n$, and $0 \neq c_{n+1} \in C_{t\left(\beta_{n}\right)}$.

By the above definition, the zero 0 is not a $\mathcal{C}$-path.

Let $V$ be the $k$-linear space spanned by all $\mathcal{C}$-paths. Let $W$ be the subspace generated by all elements of the form

$c_{1} \beta_{1} \cdots \beta_{j-1}\left(\sum_{l=1}^{m} k_{l} c_{j}^{l}\right) \beta_{j} c_{j+1} \cdots c_{n} \beta_{n} c_{n+1}-\sum_{l=1}^{m} k_{l} c_{1} \beta_{1} \cdots \beta_{j-1} c_{j}^{l} \beta_{j} c_{j+1} \cdots c_{n} \beta_{n} c_{n+1}$, where $\beta_{1} \beta_{2} \cdots \beta_{n}$ is a path in $Q, c_{i} \in C_{s\left(\beta_{i}\right)}, c_{n+1} \in C_{t\left(\beta_{n}\right)}$ for $i=1, \cdots, n$, and $k_{l} \in k, c_{j}^{l} \in C_{s\left(\beta_{j}\right)}$ for $l=1, \cdots, m$.

Let $R=V / W$ the quotient of $V$ by $W$.

Define the comultiplication $\Delta$ and the counit $\varepsilon$ of $R$ as follows:

Given a $\mathcal{C}$-path $X=c_{1} \beta_{1} c_{2} \beta_{2} \cdots c_{n} \beta_{n} c_{n+1}$, define

$$
\begin{gathered}
\triangle(X)=\sum_{i=1}^{n+1} \sum_{\left(c_{i}\right)} c_{1} \beta_{1} \cdots c_{i-1} \beta_{i-1} c_{i}^{\prime} \otimes c_{i}^{\prime \prime} \beta_{i} c_{i+1} \cdots c_{n} \beta_{n} c_{n+1}, \\
\varepsilon(X)= \begin{cases}0, & \text { if the length of } X \text { is } n>0 \\
\varepsilon_{i}(X), & \text { if } X \in C_{i} \text { for some } v_{i} \in Q_{0}\end{cases}
\end{gathered}
$$

where $\sum_{\left(c_{i}\right)} c_{i}^{\prime} \otimes c_{i}^{\prime \prime}=\triangle_{i}\left(c_{i}\right)$.

It is easy to check that the above comultiplication $\Delta$ and counit $\varepsilon$ of $R$ is well-defined and endows $R$ with a $k$-coalgebra structure. This coalgebra is called the $\mathcal{C}$-path coalgebra of $Q$ and we denote it by $R=k\left(Q^{c}, \mathcal{C}\right)$. Clearly, $k\left(Q^{c}, \mathcal{C}\right)$ is a graded coalgebra with length grading, i.e. $k\left(Q^{c}, \mathcal{C}\right)=k\left(Q_{0}, \mathcal{C}\right) \oplus k\left(Q_{1}, \mathcal{C}\right) \oplus$ $k\left(Q_{2}, \mathcal{C}\right) \oplus \cdots \oplus k\left(Q_{i}, \mathcal{C}\right) \oplus \cdots$, where $k\left(Q_{i}, \mathcal{C}\right)$ denotes the subspace generated by all $\mathcal{C}$-paths of length $i$ in $k\left(Q^{c}, \mathcal{C}\right)$ and $\triangle\left(k\left(Q_{n}, \mathcal{C}\right)\right) \subseteq \sum_{i=0}^{n} k\left(Q_{i}, \mathcal{C}\right) \otimes k\left(Q_{n-i}, \mathcal{C}\right)$. 
Observe that if $C_{i}=k$ for all $i \in Q_{0}$, then the coalgebra $k\left(Q^{c}, \mathcal{C}\right)$ defined above is the usual path coalgebra $k Q^{c}$ of $Q$, since $k Q^{c}$ is defined as the $k$-space $k Q$ with comultiplication $\Delta(p)=\sum_{\alpha \beta=p} \alpha \otimes \beta$ and counit $\varepsilon(p)=\left\{\begin{array}{l}1, \text { if the length of } p \text { is } 0 \\ 0, \text { otherwise }\end{array}\right.$ for path $p$ in $Q$. Therefore, in general, we call such coalgebra $k\left(Q^{c}, \mathcal{C}\right)$ a generalized path coalgebra when there is no ambiguity on $Q$ and $\mathcal{C}$.

Let $k\left(Q^{c}, \mathcal{C}\right)$ be a generalized path coalgebra with $\mathcal{C}=\left\{C_{i}: v_{i} \in Q_{0}\right\}$. If each $C_{i}$ is a simple coalgebra, then $k\left(Q^{c}, \mathcal{C}\right)$ is called a normal generalized path coalgebra. In this paper, all generalized path coalgebras are assumed to be normal with a finite quiver $Q$.

Since Theorem 2.2, the main result of this paper, gives the condition for a generalized path coalgebra to be a Hopf algebra, it can be thought as a generalization of the result about path coalgebras in [1] given by C.Cibils and M.Rosso.

Let $G$ be a finite group and $\mathcal{D}$ be the set of all conjugacy classes of $G$. Recall from [1] that the ramification data $r$ of $G$ is an element $r=\left(r_{D}\right)_{D \in \mathcal{D}}$ of the product set $\mathbf{N}^{\mathcal{D}}$. The Hopf quiver $Q=Q(G, r)$ corresponding to $(G, r)$ is defined as follows:

(i) $Q_{0}=\left\{v_{g}\right\}_{g \in G}$;

(ii) For any $x, y \in G$, if $y x^{-1} \in D \in \mathcal{D}$, then there are $r_{D}$ arrows from $v_{x}$ to $v_{y}$.

Note that, if $d$ and $d^{\prime}$ are conjugate in $G$, then the number of arrows from $v_{x}$ to $v_{d x}$ is equal to that of from $v_{x}$ to $v_{d^{\prime} x}$.

The path coalgebra $k Q^{c}=(k Q, \Delta, \epsilon)$ is the underlying $k$-linear space $k Q$ with the comultiplication $\Delta$ and the counit $\varepsilon$ given by

$$
\Delta(p)=\Sigma_{\beta \alpha=p} \beta \otimes \alpha \text { and } \varepsilon(p)=\left\{\begin{array}{l}
1, \text { if } p \text { is a vertex } \\
0, \text { otherwise }
\end{array}\right.
$$

for any path $p$ in $Q$.

We know from [1] the important result:

Theorem 1.1. [1] Let $Q$ be a quiver. Then the following statements are equivalent: 
(i) $Q$ is a Hopf quiver of some $(G, r)$;

(ii) $Q_{0}$ is a group $G$ and $k Q_{1}$ has a $k G$-Hopf bimodule structure with comodule maps $\delta_{L}(\alpha)=s(\alpha) \otimes \alpha$ and $\delta_{R}(\alpha)=\alpha \otimes t(\alpha)$ for $\alpha \in Q_{1}$;

(iii) the path coalgebra $k Q^{c}$ admits a graded Hopf algebra structure with length grading.

\section{Some notions and the main result}

Firstly, it is important to give an alternative definition for $\mathcal{C}$-path coalgebra through cotensor coalgebra.

Let $C$ be a coalgebra and $M \in{ }^{C} \mathcal{M}^{C}$ a $C$-bicomodule with left (resp. right) comodule structure map $\delta_{L}^{M}$ (resp. $\left.\delta_{R}^{M}\right)$. If $N$ is another $C$-bimodule, recall that the cotensor product $M \diamond_{C} N$ is the kernel of $i d \otimes \delta_{L}^{N}-\delta_{R}^{M} \otimes i d: M \otimes N \rightarrow M \otimes C \otimes$ $N$. Here the tensor product is taken over $k$. Note that $M \diamond_{C} N$ is a $C$-subbimodule of $M \otimes N$ with structure maps $\delta_{L}^{M \diamond_{C} N}=i d \otimes \delta_{L}^{N}$ and $\delta_{R}^{M \diamond_{C} N}=\delta_{R}^{M} \otimes i d$. Since $M \diamond_{C} N \subseteq M \otimes N$, we still write an element in $M \diamond_{C} N$ as $\sum_{i} m_{i} \otimes n_{i}$ in $M \otimes N$.

Set $\operatorname{CoT}_{C}(M)=\bigoplus_{n \geq 0} M^{\diamond n}$ with $M^{\diamond 0}=C, M^{\diamond 1}=M, M^{\diamond 2}=M \diamond_{C} M$, and $M^{\diamond n}$ defined inductively. Define the counit $\varepsilon$ on $\operatorname{CoT}_{C}(M)$ by $\left.\varepsilon\right|_{C}=\varepsilon_{C}$, $\left.\varepsilon\right|_{M \diamond n}=0$ for $n \geq 1$. Define the comultiplication $\triangle$ on $\operatorname{CoT}_{C}(M)$ by $\left.\triangle\right|_{C}=$ $\triangle_{C}, \triangle_{M}=\delta_{L}^{M}+\delta_{R}^{M}$. In general, for $m_{1} \otimes \cdots \otimes m_{n} \in M^{\diamond n}$ with $n \geq 2$, define

$\triangle\left(m_{1} \otimes \cdots \otimes m_{n}\right)=$

$\delta_{L}^{M}\left(m_{1}\right) \otimes m_{2} \otimes \cdots \otimes m_{n}+m_{1} \otimes\left(m_{2} \otimes \cdots \otimes m_{n}\right)+\cdots+\left(m_{1} \otimes \cdots \otimes m_{n-1}\right) \otimes$ $m_{n}+m_{1} \otimes \cdots \otimes m_{n-1} \otimes \delta_{R}^{M}\left(m_{n}\right)$

$\in C \otimes M^{\diamond n} \oplus M \otimes M^{\diamond n-1} \oplus \cdots \oplus M^{\diamond n-1} \otimes M \oplus M^{\diamond n} \otimes C \subseteq \operatorname{CoT}_{C}(M) \otimes \operatorname{CoT}_{C}(M)$.

With such structure maps $\triangle$ and $\varepsilon, \operatorname{CoT}_{C}(M)$ is a coalgebra (see [8]) which is called the cotensor coalgebra of the bicomodule $M$ over $C$.

For any $x \in k\left(Q^{c}, \mathcal{C}\right)$, write

$$
\triangle(x)=\sum_{(x)} x^{\prime} \otimes x^{\prime \prime}=\sum_{i \geq 0} \sum_{(x)}\left(x^{\prime}\right)_{i} \otimes x^{\prime \prime}=\sum_{j \geq 0} \sum_{(x)} x^{\prime} \otimes\left(x^{\prime \prime}\right)_{j}
$$

where $\sum_{(x)}\left(x^{\prime}\right)_{i} \otimes x^{\prime \prime}$ denotes the sum of all summands in $\sum_{(x)} x^{\prime} \otimes x^{\prime \prime}$ with $\left(x^{\prime}\right)_{i} \in k\left(Q_{i}, \mathcal{C}\right)$ is of length $i$; similarly, $\sum_{(x)} x^{\prime} \otimes\left(x^{\prime \prime}\right)_{j}$ is defined. 
Proposition 2.1. (see [5]) For a normal $\mathcal{C}$-path coalgebra $k\left(Q^{c}, \mathcal{C}\right)$,

(i) $k\left(Q_{0}, \mathcal{C}\right)=\sum_{v_{i} \in Q_{0}} C_{i}$ is the coradical of $k\left(Q^{c}, \mathcal{C}\right)$ as coalgebra;

(ii) $k\left(Q_{n}, \mathcal{C}\right)$ is a $k\left(Q_{0}, \mathcal{C}\right)$-bicomodule via left comodule structure map $\delta_{L}$ and right comodule structure map $\delta_{R}$ given by $\delta_{L}(x):=\sum_{(x)}\left(x^{\prime}\right)_{0} \otimes x^{\prime \prime}$ and $\delta_{R}(x):=$ $\sum_{(x)} x^{\prime} \otimes\left(x^{\prime \prime}\right)_{0}$ for $x \in k\left(Q_{n}, \mathcal{C}\right), n \geq 0$;

(iii) $k\left(Q^{c}, \mathcal{C}\right) \cong \operatorname{CoT}_{k\left(Q_{0}, \mathcal{C}\right)}\left(k\left(Q_{1}, \mathcal{C}\right)\right)$ as coalgebras.

Note that by the definition in Page 2, a $\mathcal{C}$-path is always non-zero. We call $\beta_{1} \cdots \beta_{n}(n \geq 1)$ the related usual path of a $\mathcal{C}$-path $c_{1} \beta_{1} c_{2} \beta_{2} \cdots c_{n} \beta_{n} c_{n+1}$ and write

$$
P\left(c_{1} \beta_{1} c_{2} \beta_{2} \cdots c_{n} \beta_{n} c_{n+1}\right)=\beta_{1} \cdots \beta_{n} .
$$

And, we call the trivial path $v_{i}$ in $Q$ the related usual path of a $\mathcal{C}$-path $c_{i} \in C_{i}$ for each $i$, and write $P\left(c_{i}\right)=v_{i}$. Then, $P$ is a map from the $\mathcal{C}$-path set to the path set in $Q$.

In $k\left(Q^{c}, \mathcal{C}\right)$, any non-zero element can be written as $\sum_{i=1}^{s} \sum_{j=1}^{r_{i}} X_{i j}$, where all $X_{i j}$ are $\mathcal{C}$-paths and for a fixed $i, \sum_{j=1}^{r_{i}} X_{i j}$ is assumed to be non-zero and $P\left(X_{i j_{1}}\right)=P\left(X_{i j_{2}}\right)$ for any $j_{1} \neq j_{2}$, and $P\left(X_{i_{1} j}\right) \neq P\left(X_{i_{2} l}\right)$ when $i_{1} \neq i_{2}$ for any $j, l$.

Due to this presentation, we can extend $P$ to a map $\widetilde{P}: k\left(Q^{c}, \mathcal{C}\right) \rightarrow k Q^{c}$ by setting

$$
\widetilde{P}(0)=0, \quad \widetilde{P}\left(\sum_{i=1}^{s} \sum_{j=1}^{r_{i}} X_{i j}\right)=\sum_{i=1}^{s} \widetilde{P}\left(\sum_{j=1}^{r_{i}} X_{i j}\right)=\sum_{i=1}^{s} P\left(X_{i 1}\right)
$$

for a non-zero element $\sum_{i=1}^{s} \sum_{j=1}^{r_{i}} X_{i j} \in k\left(Q^{c}, \mathcal{C}\right)$ as presented in the above paragraph, where $\widetilde{P}\left(\sum_{j=1}^{r_{i}} X_{i j}\right)$ is defined as $P\left(X_{i 1}\right)$ for any $i$.

Then, this map $\widetilde{P}: k\left(Q^{c}, \mathcal{C}\right) \rightarrow k Q^{c}$ is well-defined. Note that $\widetilde{P}$ is neither $k$-linear nor surjective, since every non-zero element of the image $\operatorname{Im} \widetilde{P}$ needs to be a sum of some distinct paths in $Q$.

For example, let $Q$ be the Kronecker quiver $\bullet \frac{\alpha}{\beta} \bullet \bullet$ and $C_{1}, C_{2}$ be simple coalgebras of dimension greater than 1 . Then, for any nonzero $c_{1}, d_{1}, c_{1}^{\prime}, d_{1}^{\prime} \in C_{1}$, $c_{2}, d_{2}, c_{2}^{\prime}, d_{2}^{\prime} \in C_{2}$ with $\left(c_{1}, c_{1}^{\prime}\right) \neq-\left(c_{2}, c_{2}^{\prime}\right),\left(d_{1}, d_{1}^{\prime}\right) \neq-\left(d_{2}, d_{2}^{\prime}\right)$ as elements in the 
$k$-linear space $C_{1} \times C_{2}$, we have

$$
\widetilde{P}\left(c_{1} \alpha c_{1}^{\prime}+c_{2} \alpha c_{2}^{\prime}+d_{1} \beta d_{1}^{\prime}+d_{2} \beta d_{2}^{\prime}\right)=\alpha+\beta
$$

Definition 2.1. Suppose $*: k\left(Q^{c}, \mathcal{C}\right) \times k\left(Q^{c}, \mathcal{C}\right) \rightarrow k\left(Q^{c}, \mathcal{C}\right)$ is a partial binary operation. For any $X, X^{\prime}, Y, Y^{\prime} \in k\left(Q^{c}, \mathcal{C}\right)$, if $\widetilde{P}(X)=\widetilde{P}\left(X^{\prime}\right)$ and $\widetilde{P}(Y)=$ $\widetilde{P}\left(Y^{\prime}\right)$ imply $\widetilde{P}(X * Y)=\widetilde{P}\left(X^{\prime} * Y^{\prime}\right)$, then $k\left(Q^{c}, \mathcal{C}\right)$ is called $\mathcal{C}$-arrow-stable under the operation $*$.

In this definition, that $*$ is partial implies the domain of $*$ may be a proper subset of $k\left(Q^{c}, \mathcal{C}\right) \times k\left(Q^{c}, \mathcal{C}\right)$.

For example, assume that $k\left(Q_{0}, \mathcal{C}\right)$ has an algebra structure and $k\left(Q_{1}, \mathcal{C}\right)$ is a $k\left(Q_{0}, \mathcal{C}\right)$-module under some action $\cdot$ For $c_{i} \in C_{i}, c_{j} \in C_{j}, c_{k} \in C_{k}$, and an arrow $\alpha$ in $Q_{1}$ from $v_{j}$ to $v_{k}$, assume

$$
c_{i} \cdot\left(c_{j} \alpha c_{k}\right)=\sum_{l=1}^{m} d_{s_{l}} \beta_{l} d_{t_{l}} \neq 0,
$$

where each $d_{s_{l}} \beta_{l} d_{t_{l}} \neq 0, d_{s_{l}} \in C_{s_{l}}, d_{t_{l}} \in C_{t_{l}}$ and $\beta_{l}$ is an arrow from $v_{s_{l}}$ to $v_{t_{l}}$ for $l=1,2 \cdots m$. Suppose for any other $0 \neq c_{i}^{\prime} \in C_{i}, 0 \neq c_{j}^{\prime} \in C_{j}, 0 \neq c_{k}^{\prime} \in C_{k}$, there exist $d_{s_{l}}^{\prime} \in C_{s_{l}}$ and $d_{t_{l}}^{\prime} \in C_{t_{l}}$ such that

$$
c_{i}^{\prime} \cdot\left(c_{j}^{\prime} \alpha c_{k}^{\prime}\right)=\sum_{l=1}^{n} d_{s_{l}}^{\prime} \beta_{l}^{\prime} d_{t_{l}}^{\prime} \neq 0,
$$

where $d_{s_{l}}^{\prime} \beta_{l}^{\prime} d_{t_{l}}^{\prime} \neq 0, \beta_{l}^{\prime}$ is an arrow from $v_{s_{l}}$ to $v_{t_{l}}$ for $l=1,2 \cdots n$, and the sets $\left\{\beta_{l}^{\prime}: l=1,2, \cdots, n\right\}=\left\{\beta_{l}: l=1,2, \cdots, m\right\}$. Then, $k\left(Q_{1}, \mathcal{C}\right)$ is $\mathcal{C}$-arrow-stable under the module action $\cdot$. Here and in the sequel, if there are several same elements in a set, we always consider them as one element. If there exist $0 \neq c_{i} \in C_{i}, 0 \neq c_{j} \in C_{j}, 0 \neq c_{k} \in C_{k}$ and an arrow $\alpha$ in $Q_{1}$ from $v_{j}$ to $v_{k}$ such that $c_{i} \cdot\left(c_{j} \alpha c_{k}\right)=0$, then the $\mathcal{C}$-arrow-stability of $k\left(Q_{1}, \mathcal{C}\right)$ under the module action - implies that for any $c_{i}^{\prime} \in C_{i}, c_{j}^{\prime} \in C_{j}, c_{k}^{\prime} \in C_{k}$, it always holds that $c_{i}^{\prime} \cdot\left(c_{j}^{\prime} \alpha c_{k}^{\prime}\right)=0$.

For another example, suppose that the generalized path coalgebra $k\left(Q^{c}, \mathcal{C}\right)$ has a Hopf algebra structure with the multiplication $\cdot$ such that the product of 
two $\mathcal{C}$-paths are always non-zero, that is,

$\left(a_{1} \beta_{1} a_{2} \beta_{2} \cdots a_{n} \beta_{n} a_{n+1}\right) \cdot\left(b_{1} \gamma_{1} b_{2} \gamma_{2} \cdots b_{m} \gamma_{m} b_{m+1}\right)=\sum_{l=1}^{t} c_{1}^{l} \delta_{1}^{l} c_{2}^{l} \delta_{2}^{l} \cdots c_{w}^{l} \delta_{w}^{l} c_{w+1}^{l} \neq 0$,

where each $c_{1}^{l} \delta_{1}^{l} c_{2}^{l} \delta_{2}^{l} \cdots c_{w}^{l} \delta_{w}^{l} c_{w+1}^{l}$ is a $\mathcal{C}$-path, $a_{p} \in C_{i_{p}}, b_{q} \in C_{j_{q}}, c_{u}^{l} \in C_{k_{u}^{l}}$ for $p=1, \cdots, n+1, q=1, \cdots, m+1, u=1, \cdots, w+1, l=1, \cdots, t$. Then, the $\mathcal{C}$ arrow-stability of $k\left(Q^{c}, \mathcal{C}\right)$ under the multiplication - implies for any $0 \neq a_{p}^{\prime} \in C_{i_{p}}$, $0 \neq b_{q}^{\prime} \in C_{j_{q}}$ for $p=1, \cdots, n+1, q=1, \cdots, m+1$, there exist $0 \neq c_{u}^{\prime l} \in C_{k_{u}^{l}}$ for $u=1, \cdots, w+1$ and $l=1, \cdots, s$ such that

$$
\left(a_{1}^{\prime} \beta_{1} a_{2}^{\prime} \beta_{2} \cdots a_{n}^{\prime} \beta_{n} a_{n+1}^{\prime}\right) \cdot\left(b_{1}^{\prime} \gamma_{1} b_{2}^{\prime} \gamma_{2} \cdots b_{m}^{\prime} \gamma_{m} b_{m+1}^{\prime}\right)=\sum_{l=1}^{s} c_{1}^{\prime l} \delta_{1}^{\prime l} c_{2}^{\prime l} \delta_{2}^{\prime l} \cdots c_{w}^{\prime l} \delta_{w}^{\prime l} c_{w+1}^{\prime l} \neq 0,
$$

where each $c_{1}^{\prime l} \delta_{1}^{l} c_{2}^{\prime l} \delta_{2}^{l} \cdots c_{w}^{\prime l} \delta_{w}^{l} c_{w+1}^{\prime l}$ is a $\mathcal{C}$-path and the sets $\left\{\delta_{1}^{\prime l} \delta_{2}^{\prime l} \cdots \delta_{w}^{\prime l}: l=\right.$ $1,2, \cdots, s\}=\left\{\delta_{1}^{l} \delta_{2}^{l} \cdots \delta_{w}^{l}: l=1,2, \cdots, t\right\}$.

It is known that when $k\left(Q^{c}, \mathcal{C}\right)$ has a graded Hopf algebra structure with length grading, the coradical $k\left(Q_{0}, \mathcal{C}\right)=\sum_{v_{i} \in Q_{0}} C_{i}$ is a Hopf subalgebra of $k\left(Q^{c}, \mathcal{C}\right)$ by the definition of grading.

Now, suppose that the coradical $k\left(Q_{0}, \mathcal{C}\right)=\sum_{v_{i} \in Q_{0}} C_{i}$ of $k\left(Q^{c}, \mathcal{C}\right)$ has a Hopf algebra structure. Since $C_{i}$ and $C_{j}$ are coalgebras, $\triangle\left(C_{i}\right) \subseteq C_{i} \otimes C_{i}$ and $\triangle\left(C_{j}\right) \subseteq C_{j} \otimes C_{j}$. Then $\triangle\left(C_{i} C_{j}\right)=\triangle\left(C_{i}\right) \triangle\left(C_{j}\right) \subseteq\left(C_{i} \otimes C_{i}\right)\left(C_{j} \otimes C_{j}\right)=$ $C_{i} C_{j} \otimes C_{i} C_{j}$. It means that $C_{i} C_{j}$ is a subcoalgebra of the coradical $k\left(Q_{0}, \mathcal{C}\right)$. Since $C_{i}\left(v_{i} \in Q_{0}\right)$ are all the simple subcoalgebras $k\left(Q^{c}, \mathcal{C}\right)$, it is easy to see that there exist $v_{k_{1}}, v_{k_{2}}, \cdots, v_{k_{p}} \in Q_{0}$ such that $C_{i} C_{j}=C_{k_{1}}+C_{k_{2}}+\cdots+C_{k_{p}}$ if $C_{i} C_{j} \neq 0$.

According to these statements, we introduce the following:

Definition 2.2. The coradical $k\left(Q_{0}, \mathcal{C}\right)$ of $k\left(Q^{c}, \mathcal{C}\right)$ is said to be $Q_{0}$-closed if $k\left(Q_{0}, \mathcal{C}\right)$ has a Hopf algebra structure satisfying

(i) for any $v_{i}, v_{j} \in Q_{0}$ and any $0 \neq c_{i} \in C_{i}, 0 \neq c_{j} \in C_{j}$, there exists uniquely a $v_{k} \in Q_{0}$ such that $0 \neq c_{i} c_{j} \in C_{k}$; and

(ii) for any $v_{i}, v_{j}, v_{k} \in Q_{0}$, and any $0 \neq c_{i} \in C_{i}, 0 \neq c_{j} \in C_{j}, 0 \neq c_{k} \in C_{k}$, it holds that $\triangle\left(c_{i}\right)\left(c_{j} \otimes c_{k}\right) \neq 0$ and $\left(c_{j} \otimes c_{k}\right) \triangle\left(c_{i}\right) \neq 0$. 
Definition 2.3. Let $G$ be a group, the coradical $k\left(Q_{0}, \mathcal{C}\right)$ of $k\left(Q^{c}, \mathcal{C}\right)$ is said to be of $G$-type if $Q_{0}=\left\{v_{g}\right\}_{g \in G}$ and $k\left(Q_{0}, \mathcal{C}\right)$ has a Hopf algebra structure satisfying

(i) for any $v_{i}, v_{j} \in Q_{0}$ and any $0 \neq c_{i} \in C_{i}, 0 \neq c_{j} \in C_{j}$, it holds that $0 \neq c_{i} c_{j} \in C_{i j}$;

(ii) for any $v_{i}, v_{j}, v_{k} \in Q_{0}$, and any $0 \neq c_{i} \in C_{i}, 0 \neq c_{j} \in C_{j}, 0 \neq c_{k} \in C_{k}$, it holds that $\triangle\left(c_{i}\right)\left(c_{j} \otimes c_{k}\right) \neq 0$ and $\left(c_{j} \otimes c_{k}\right) \triangle\left(c_{i}\right) \neq 0$.

Note that Definition 2.2 (ii) is the same to Definition 2.3 (ii). Definition 2.2 (i) implies $C_{i} C_{j}=C_{k}$ and Definition 2.3 (i) implies $C_{i} C_{j}=C_{i j}$. Trivially, when $k\left(Q_{0}, \mathcal{C}\right)$ is of $G$-type, it must be $Q_{0}$-closed; but the converse is not true in general.

We can now state the main result of this paper with proof given in the next section.

Theorem 2.2. Let $k\left(Q^{c}, \mathcal{C}\right)$ be a normal generalized path coalgebra over a field $k$. Then the following statements are equivalent:

(i) $Q$ is a Hopf quiver of some $(G, r)$ such that $k\left(Q_{0}, \mathcal{C}\right)$ is of $G$-type;

(ii) $k\left(Q_{0}, \mathcal{C}\right)$ is $Q_{0}$-closed such that $k\left(Q_{1}, \mathcal{C}\right)$ has a $k\left(Q_{0}, \mathcal{C}\right)$-Hopf bimodule structure with $\mathcal{C}$-arrow-stable module structure and with the comodule structure maps

$$
\delta_{L}(x):=\sum_{(x)}\left(x^{\prime}\right)_{0} \otimes x^{\prime \prime} \quad \text { and } \quad \delta_{R}(x):=\sum_{(x)} x^{\prime} \otimes\left(x^{\prime \prime}\right)_{0}
$$

for $x \in k\left(Q_{1}, \mathcal{C}\right)$;

(iii) $k\left(Q^{c}, \mathcal{C}\right)$ admits a graded Hopf algebra structure with length grading, $Q_{0}$ closed coradical and $\mathcal{C}$-arrow-stable multiplication.

Due to this result, we get a Hopf algebra structure $H$ on $k\left(Q^{c}, \mathcal{C}\right)$ if any one of three statements holds. In particular, when $Q$ is a finite acyclic quiver and all $C_{i}$ are finite-dimensional with $\operatorname{dim}_{k} C_{j}>1$ for at least one $j, H$ is a finite-dimensional non-pointed Hopf algebra. 


\section{Proof of the main result}

Take a coalgebra $C$ and $c \in C$, write $\triangle(c)=\sum d_{i} \otimes e_{i}$. This representation is not unique. However, if one takes the $e_{i}$ 's linearly independent, the subspace spanned by the $d_{i}$ 's is independent of the representation. Call it $L c$. Define $R c$ analogously. Moreover, for a subset $X \subseteq C$, define $L(X)=\sum_{c \in X} L c$ and $R(X)=\sum_{c \in X} R c$.

In [3], we have known:

Lemma 3.1. (see [3]) For a coalgebra $C$ and $c \in C$, it holds that $R R c=R c$, $L L c=L c, L R c=R L c$.

It is easy to check that $L(c+d) \subseteq L c+L d$ and $R(c+d) \subseteq R c+R d$ for any $c, d \in C$. Thus, we have:

Lemma 3.2. Assume that $C$ is a coalgebra. Then,

(i) a k-subspace $D$ of $C$ is a subcoalgebra if and only if $L(D) \subseteq D$ and $R(D) \subseteq D$

(ii) $L(C)$ and $R(C)$ are subcoalgebras of $C$;

(iii) $L(C)=C=R(C)$ when $C$ is a simple coalgebra.

Proof. (i) ( $\Longrightarrow)$ : It is trivial from the definitions of $L(D)$ and $R(D)$.

$(\Longleftarrow)$ : For any $d \in D$, let $\Delta(d)=\sum_{i=1}^{t} d_{i}^{\prime} \otimes d_{i}^{\prime \prime}$ be the shortest representation of $\Delta(d)$, then $\left\{d_{i}^{\prime}\right\}_{i=1, \cdots, t}$ and $\left\{d_{i}^{\prime \prime}\right\}_{i=1, \cdots, t}$ are both $k$-linearly independent. Thus, $L d$ is generated by $\left\{d_{i}^{\prime}\right\}_{i=1, \cdots, t}$ and $R d$ is generated by $\left\{d_{i}^{\prime \prime}\right\}_{i=1, \cdots, t}$. When $L(D) \subseteq$ $D$ and $R(D) \subseteq D$, we have $d_{i}^{\prime} \in L d \subseteq L(D) \subseteq D$ and $d_{i}^{\prime \prime} \in R d \subseteq R(D) \subseteq D$ for $i=1,2, \cdots, t$. So $D$ is a subcoalgebra.

(ii) By Lemma 3.1, $L(L(C))=L\left(\sum_{c \in C} L c\right) \subseteq \sum_{c \in C} L L c=\sum_{c \in C} L c=L(C)$. And, $R(L(C))=R\left(\sum_{c \in C} L c\right) \subseteq \sum_{c \in C} R L c=\sum_{c \in C} L R c \subseteq \sum_{c \in C} L c=L(C)$, where the second " $\subseteq$ " is from the $R c \subseteq C$ for any $c \in C$.

Similarly, $L(R(C)) \subseteq R(C), R(R(C)) \subseteq R(C)$. Thus, by (i), this result holds.

(iii) It is obvious due to (ii) and the simplicity of $C$. 
Proposition 3.3. Suppose $B$ is a Hopf algebra with antipode $S$. Let $D$ be a simple subcoalgebra of $(B, \Delta)$. Then $S(D)$ is a simple subcoalgebra of $\left(B, \Delta^{o p}\right)$.

Proof. It is known that $S$ is a coalgebra homomorphism from $(B, \Delta)$ to $\left(B, \Delta^{o p}\right)$. Note that the inverse image and image of a subcoalgebra is also a subcoalgebra. It is easy to see $S(D) \neq 0$ always holds. So, $S(D)$ is a simple subcoalgebra of $\left(B, \Delta^{o p}\right)$.

Proposition 3.4. Suppose that $k\left(Q^{c}, \mathcal{C}\right)$ has a graded Hopf algebra structure with length grading and $Q_{0}$-closed coradical. Then the index set of $Q_{0}$ possesses a group structure, denoted as $(G, \cdot)$, such that $k\left(Q_{0}, \mathcal{C}\right)$ is of $G$-type.

Proof. Since $k\left(Q^{c}, \mathcal{C}\right)$ is a graded Hopf algebra with length grading, the coradical $k\left(Q_{0}, \mathcal{C}\right)=\sum_{v_{i} \in Q_{0}} C_{i}$ is a finite dimensional Hopf subalgebra of $k\left(Q^{c}, \mathcal{C}\right)$.

From the $Q_{0}$-closure of $k\left(Q_{0}, \mathcal{C}\right)$, for any $v_{i}, v_{j} \in Q_{0}$, there exists uniquely a $v_{k} \in Q_{0}$ such that $C_{i} C_{j}=C_{k}$. Then we define the multiplication $\cdot$ by $i \cdot j=$ $k$. This multiplication - is associative, since the multiplication of $k\left(Q^{c}, \mathcal{C}\right)$ is associative.

For the identity 1 of $k\left(Q^{c}, \mathcal{C}\right), k 1$ is the simple subcoalgebra containing 1. Then there exists $v_{e} \in Q_{0}$ with $C_{e}=k 1$.

Suppose $S$ is the antipode of $k\left(Q^{c}, \mathcal{C}\right)$. Then, the restriction $S_{0}$ of $S$ on $k\left(Q_{0}, \mathcal{C}\right)$ is the antipode of $k\left(Q_{0}, \mathcal{C}\right)$ and is invertible on $k\left(Q_{0}, \mathcal{C}\right)$. Then, $S\left(C_{i}\right) \neq$ 0. Thus, by Proposition 3.3, $S\left(C_{i}\right)$ is simple. Then there is $v_{i^{\prime}} \in Q_{0}$ such that $S\left(C_{i}\right)=C_{i^{\prime}}$.

Since the coradical $k\left(Q_{0}, \mathcal{C}\right)$ is $Q_{0}$-closed, there is $v_{k} \in Q_{0}$ with $C_{i} C_{i^{\prime}}=C_{k}$. And, $C_{i} C_{i^{\prime}}=C_{i} S\left(C_{i}\right) \supseteq(i d * S)\left(C_{i}\right)=\eta \varepsilon\left(C_{i}\right)=\varepsilon\left(C_{i}\right) 1=C_{e}$ since trivially $\varepsilon\left(C_{i}\right) \neq 0$. However, $C_{k}$ is simple, so we get $C_{e}=C_{k}$. Thus, $C_{i} C_{i^{\prime}}=C_{e}$. Similarly $C_{i^{\prime}} C_{i}=C_{e}$. Obviously, $C_{i} C_{e}=C_{i}=C_{e} C_{i}$ for $v_{i} \in Q_{0}$. By the definition of the multiplication $\cdot$, it follows that $i \cdot i^{\prime}=e=i^{\prime} \cdot i$ and $i \cdot e=e \cdot i=i$. Therefore, the index set of $Q_{0}$ becomes into a group with identity $e$ and the inverse $i^{-1}=i^{\prime}$ for any $v_{i} \in Q_{0}$. Denote this group by $(G, \cdot)$. Then, $k\left(Q_{0}, \mathcal{C}\right)$ is of $G$-type. 
Proposition 3.5. Suppose $k\left(Q^{c}, \mathcal{C}\right)$ has a graded Hopf algebra structure with length grading, $Q_{0}$-closed coradical and $\mathcal{C}$-arrow-stable multiplication. Then $k Q^{c}$ also has a graded Hopf algebra structure with length grading.

Proof. We need to define a multiplication such that the comultiplication $\Delta$ and the counit $\varepsilon$ on $k Q^{c}$ are algebra homomorphisms.

Firstly from Proposition 3.4, the index set of $Q_{0}$ has a group structure, denoted by $(G, \cdot)$. Then for any $v_{i}, v_{j} \in Q_{0}$, define $v_{i} v_{j}=v_{i \cdot j}$.

For any paths $\beta_{1} \beta_{2} \cdots \beta_{n}$ and $\gamma_{1} \gamma_{2} \cdots \gamma_{m}$ in $k Q^{c}$ with $m+n \geq 1$, define

$$
\left(\beta_{1} \beta_{2} \cdots \beta_{n}\right) \cdot\left(\gamma_{1} \gamma_{2} \cdots \gamma_{m}\right)=0
$$

if there are two $\mathcal{C}$-paths $a_{1} \beta_{1} a_{2} \beta_{2} \cdots a_{n} \beta_{n} a_{n+1}$ and $b_{1} \gamma_{1} b_{2} \gamma_{2} \cdots b_{m} \gamma_{m} b_{m+1}$ in $k\left(Q^{c}, \mathcal{C}\right)$ such that

$$
\left(a_{1} \beta_{1} a_{2} \beta_{2} \cdots a_{n} \beta_{n} a_{n+1}\right) \cdot\left(b_{1} \gamma_{1} b_{2} \gamma_{2} \cdots b_{m} \gamma_{m} b_{m+1}\right)=0 .
$$

Otherwise, define

$$
\left(\beta_{1} \beta_{2} \cdots \beta_{n}\right) \cdot\left(\gamma_{1} \gamma_{2} \cdots \gamma_{m}\right)=\sum_{i=1}^{s} P\left(X_{i 1}\right)
$$

if there are two $\mathcal{C}$-paths $a_{1} \beta_{1} a_{2} \beta_{2} \cdots a_{n} \beta_{n} a_{n+1}$ and $b_{1} \gamma_{1} b_{2} \gamma_{2} \cdots b_{m} \gamma_{m} b_{m+1}$ in $k\left(Q^{c}, \mathcal{C}\right)$ such that

$$
\left(a_{1} \beta_{1} a_{2} \beta_{2} \cdots a_{n} \beta_{n} a_{n+1}\right) \cdot\left(b_{1} \gamma_{1} b_{2} \gamma_{2} \cdots b_{m} \gamma_{m} b_{m+1}\right)=\sum_{i=1}^{s} \sum_{j=1}^{r_{i}} X_{i j} \neq 0
$$

where each $X_{i j}$ is a $\mathcal{C}$-path, $\sum_{j}^{r_{i}} X_{i j} \neq 0$ is the sum of some $\mathcal{C}$-paths whose related usual paths are the same for any $i$, and $P\left(X_{i_{1} j_{1}}\right) \neq P\left(X_{i_{2} j_{2}}\right)$ when $i_{1} \neq i_{2}$.

This multiplication is well-defined since the multiplication in $k\left(Q^{c}, \mathcal{C}\right)$ is $\mathcal{C}$ arrow-stable. Its associative law and distributive law follow from that of $k\left(Q^{c}, \mathcal{C}\right)$. Then $k Q^{c}$ is a $k$-algebra. Since the multiplication in $k\left(Q^{c}, \mathcal{C}\right)$ is length-graded, this multiplication in $k Q^{c}$ is also length-graded.

It is known $k\left(Q^{c}, \mathcal{C}\right)$ is a Hopf algebra, so the comultiplication $\triangle$ and the counit $\varepsilon$ are algebra homomorphisms, i.e. 


$$
\begin{aligned}
& \triangle\left(\left(a_{1} \beta_{1} a_{2} \beta_{2} \cdots a_{n} \beta_{n} a_{n+1}\right) \cdot\left(b_{1} \gamma_{1} b_{2} \gamma_{2} \cdots b_{m} \gamma_{m} b_{m+1}\right)\right) \\
= & \triangle\left(a_{1} \beta_{1} a_{2} \beta_{2} \cdots a_{n} \beta_{n} a_{n+1}\right) \cdot \triangle\left(b_{1} \gamma_{1} b_{2} \gamma_{2} \cdots b_{m} \gamma_{m} b_{m+1}\right) ; \\
& \varepsilon\left(\left(a_{1} \beta_{1} a_{2} \beta_{2} \cdots a_{n} \beta_{n} a_{n+1}\right) \cdot\left(b_{1} \gamma_{1} b_{2} \gamma_{2} \cdots b_{m} \gamma_{m} b_{m+1}\right)\right) \\
= & \varepsilon\left(a_{1} \beta_{1} a_{2} \beta_{2} \cdots a_{n} \beta_{n} a_{n+1}\right) \cdot \varepsilon\left(b_{1} \gamma_{1} b_{2} \gamma_{2} \cdots b_{m} \gamma_{m} b_{m+1}\right) .
\end{aligned}
$$

Due to their definitions, the formulae of the comultiplication $\triangle$ and the counit $\varepsilon$ in $k Q^{c}$ are the same as that in $k\left(Q^{c}, \mathcal{C}\right)$. By (5) and (6) and the $\mathcal{C}$-arrow stability of the multiplication of $k\left(Q^{c}, \mathcal{C}\right)$, it follows that

$$
\begin{gathered}
\triangle\left(\beta_{1} \beta_{2} \cdots \beta_{n} \cdot \gamma_{1} \gamma_{2} \cdots \gamma_{m}\right)=\triangle\left(\beta_{1} \beta_{2} \cdots \beta_{n}\right) \cdot \triangle\left(\gamma_{1} \gamma_{2} \cdots \gamma_{m}\right) \\
\varepsilon\left(\beta_{1} \beta_{2} \cdots \beta_{n} \cdot \gamma_{1} \gamma_{2} \cdots \gamma_{m}\right)=\varepsilon\left(\beta_{1} \beta_{2} \cdots \beta_{n}\right) \cdot \varepsilon\left(\gamma_{1} \gamma_{2} \cdots \gamma_{m}\right)
\end{gathered}
$$

in $k Q^{c}$. Therefore, $k Q^{c}$ is a Hopf algebra.

Suppose $k\left(Q^{c}, \mathcal{C}\right)$ has a graded Hopf algebra structure with length grading, $Q_{0}$-closed coradical and $\mathcal{C}$-arrow-stable multiplication. By Proposition 3.5, $k Q^{c}$ also has a graded Hopf algebra structure with length grading. Then by Theorem 1.1, the index set of $Q_{0}$ has a group structure, denoted by $(G, *)$, such that $Q$ is a Hopf quiver $Q(G, r)$ for some ramification $r$. Meanwhile by Proposition 3.4, the index set of $Q_{0}$ has another a group structure, denoted by $(G, \cdot)$, such that $k\left(Q_{0}, \mathcal{C}\right)$ is of $G$-type. In fact, since the two multiplications in the group $G$ are determined by the multiplication in the Hopf subalgebra $k\left(Q_{0}, \mathcal{C}\right)$, then these two group structures $(G, *)$ and $(G, \cdot)$ are coincided. In the summary, we obtain:

Proposition 3.6. Suppose $k\left(Q^{c}, \mathcal{C}\right)$ has a graded Hopf algebra structure with length grading, $Q_{0}$-closed coradical and $\mathcal{C}$-arrow-stable multiplication. Then $Q$ is a Hopf quiver of some $(G, r)$ such that $k\left(Q_{0}, \mathcal{C}\right)$ is of $G$-type.

Lemma 3.7. (see [10]) Let $A$ be a k-algebra and $C$ be a k-coalgebra with coradical filtration $\left\{C_{i}\right\}_{i \geq 0}$. Then for any $f \in \operatorname{Hom}_{k}(C, A), f$ is invertible in $H_{\text {om }}(C, A)$ if and only if $\left.f\right|_{C_{0}}$ is invertible in $\operatorname{Hom}_{k}\left(C_{0}, A\right)$. 
From this lemma, we can get the following:

Proposition 3.8. Let $H$ be a bialgebra. If the coradical $H_{0}$ is a Hopf algebra which is a sub-bialgebra of $H$ as bialgebra, then $H$ is a Hopf algebra.

Proof. In Lemma 3.7, let $C=A=H$ and $f=i d_{H}$. Then, $\left.f\right|_{H_{0}}=i d_{H_{0}}$ is from $H_{0}$ to $H_{0}$, also from $H_{0}$ to $H$. Since $H_{0}$ is a Hopf algebra, there exists $S \in \operatorname{Hom}_{k}\left(H_{0}, H_{0}\right) \subseteq \operatorname{Hom}_{k}\left(H_{0}, H\right)$ such that $S * i d_{H_{0}}=\eta_{H_{0}} \varepsilon_{H_{0}}$. Since the identity map of $H_{0}$ is the same as that of $H$, then $\left.S * f\right|_{H_{0}}=\eta_{H} \varepsilon_{H_{0}}$ for $\left.f\right|_{H_{0}} \in$ $\operatorname{Hom}_{k}\left(H_{0}, H\right)$. Then by Lemma 3.7, $f=i d_{H}$ is invertible in $H_{k}(H, H)$ with inverse $f^{-1}=T$. Thus, $T$ is the antipode of $H$. So, $H$ is a Hopf algebra.

Proposition 3.9. (see [1] [9]) Let $\psi_{0}: X \rightarrow C$ be a coalgebra map, and $\psi_{1}: X \rightarrow$ $M$ a C-bicomodule map. Let $\psi_{n}: X \rightarrow M^{\otimes n}$ be the composition

$$
\psi_{n}: X \stackrel{\triangle^{(n-1)}}{\rightarrow} X \otimes X \otimes \cdots \otimes X \stackrel{\psi_{1}^{\otimes n}}{\rightarrow} M^{\otimes n}, \quad n \geq 2
$$

Then $\psi_{n}$ is a C-bicomodule map with $\operatorname{Im}\left(\psi_{n}\right) \subseteq M^{\diamond n}$.

If for each $x \in X$ there are only finite $i$ such that $\psi_{i}(x) \neq 0$, then $\psi: X \rightarrow$ $\operatorname{CoT}_{C}(M)$ is a coalgebra map, where $\psi=\sum_{i \geq 0} \psi_{i}$.

Now it is ready for us to prove the main result in this paper:

Proof. of Theorem 2.2:

(i) $\Rightarrow$ (ii): $\quad$ Since $k\left(Q_{0}, \mathcal{C}\right)$ is of $G$-type, there is an algebra structure such that $k\left(Q_{0}, \mathcal{C}\right)$ is a Hopf algebra and is $Q_{0}$-closed as the coradical of $k\left(Q^{c}, \mathcal{C}\right)$. Now, we give the $k\left(Q_{0}, \mathcal{C}\right)$-Hopf bimodule structure on $k\left(Q_{1}, \mathcal{C}\right)$ depending upon the $k G$-Hopf bimodule structure on $k Q_{1}$ ( see Theorem 1.1) as follows:

(1) the left module action $\cdot$

$$
c_{i} \cdot\left(c_{j} \alpha c_{k}\right)=\sum_{\left(c_{i}\right)}\left(c_{i}^{\prime} c_{j}\right)(i \cdot \alpha)\left(c_{i}^{\prime \prime} c_{k}\right)
$$

where $c_{i} \in C_{i}, c_{j} \in C_{j}, c_{k} \in C_{k}, \alpha$ is an arrow in $Q_{1}$ from $v_{j}$ to $v_{k}$, and $i \cdot \alpha$ is the left $k G$-module action on $k Q_{1}$; 
(2) the right module action $\cdot$ :

$$
\left(c_{j} \alpha c_{k}\right) \cdot c_{i}=\sum_{\left(c_{i}\right)}\left(c_{j} c_{i}^{\prime}\right)(\alpha \cdot i)\left(c_{k} c_{i}^{\prime \prime}\right)
$$

where $c_{i} \in C_{i}, c_{j} \in C_{j}, c_{k} \in C_{k}, \alpha$ is an arrow in $Q_{1}$ from $v_{j}$ to $v_{k}$, and $\alpha \cdot i$ is the right $k G$-module action on $k Q_{1}$;

(3) the left comodule coaction $\delta_{L}$ :

$$
\delta_{L}\left(c_{j} \alpha c_{k}\right)=\sum_{\left(c_{j}\right)} c_{j}^{\prime} \otimes c_{j}^{\prime \prime} \alpha c_{k}
$$

where $c_{j} \in C_{j}, c_{k} \in C_{k}, \alpha$ is an arrow in $Q_{1}$ from $v_{j}$ to $v_{k}$;

(4) the right comodule coaction $\delta_{R}$ :

$$
\delta_{R}\left(c_{j} \alpha c_{k}\right)=\sum_{\left(c_{j}\right)} c_{j} \alpha c_{k}^{\prime} \otimes c_{k}^{\prime \prime}
$$

where $c_{j} \in C_{j}, c_{k} \in C_{k}, \alpha$ is an arrow in $Q_{1}$ from $v_{j}$ to $v_{k}$.

It is trivial that $k\left(Q_{1}, \mathcal{C}\right)$ is a $k\left(Q_{0}, \mathcal{C}\right)$-bimodule, since the module action is based on the $k G$-module action on $k Q_{1}$. And $k\left(Q_{1}, \mathcal{C}\right)$ is also a $k\left(Q_{0}, \mathcal{C}\right)$ bicomodule by Proposition 2.1 (ii). Moreover, $\delta_{L}$ and $\delta_{R}$ are both $k\left(Q_{0}, \mathcal{C}\right)$ bimodule homomorphisms. In fact, for any $c_{i} \in C_{i}, c_{j} \in C_{j}, c_{k} \in C_{k}$, and any arrow $\alpha$ in $Q_{1}$ from $v_{j}$ to $v_{k}$,

$$
\begin{aligned}
\delta_{L}\left(c_{i} \cdot\left(c_{j} \alpha c_{k}\right)\right) & =\delta_{L}\left(\sum_{\left(c_{i}\right)}\left(c_{i}^{\prime} c_{j}\right)(i \cdot \alpha)\left(c_{i}^{\prime \prime} c_{k}\right)\right) \\
& =\sum_{\left(c_{i}\right)\left(c_{j}\right)} c_{i}^{\prime} c_{j}^{\prime} \otimes c_{i}^{\prime \prime} c_{j}^{\prime \prime}(i \cdot \alpha) c_{i}^{\prime \prime \prime} c_{k}, \\
c_{i} \cdot \delta_{L}\left(c_{j} \alpha c_{k}\right) & =c_{i} \cdot\left(\sum_{\left(c_{j}\right)} c_{j}^{\prime} \otimes c_{j}^{\prime \prime} \alpha c_{k}\right) \\
& =\sum_{\left(c_{i}\right)\left(c_{j}\right)} c_{i}^{\prime} c_{j}^{\prime} \otimes c_{i}^{\prime \prime} \cdot\left(c_{j}^{\prime \prime} \alpha c_{k}\right) \\
& =\sum_{\left(c_{i}\right)\left(c_{j}\right)} c_{i}^{\prime} c_{j}^{\prime} \otimes c_{i}^{\prime \prime} c_{j}^{\prime \prime}(i \cdot \alpha) c_{i}^{\prime \prime \prime} c_{k},
\end{aligned}
$$


then $\delta_{L}\left(c_{i} \cdot\left(c_{j} \alpha c_{k}\right)\right)=c_{i} \cdot \delta_{L}\left(c_{j} \alpha c_{k}\right)$.

$$
\begin{aligned}
\delta_{L}\left(\left(c_{j} \alpha c_{k}\right) \cdot c_{i}\right) & =\delta_{L}\left(\sum_{\left(c_{i}\right)}\left(c_{j} c_{i}^{\prime}\right)(\alpha \cdot i)\left(c_{k} c_{i}^{\prime \prime}\right)\right) \\
& =\sum_{\left(c_{i}\right)\left(c_{j}\right)} c_{j}^{\prime} c_{i}^{\prime} \otimes c_{j}^{\prime \prime} c_{i}^{\prime \prime}(\alpha \cdot i) c_{k} c_{i}^{\prime \prime \prime} \\
\delta_{L}\left(c_{j} \alpha c_{k}\right) \cdot c_{i} & =\left(\sum_{\left(c_{j}\right)} c_{j}^{\prime} \otimes c_{j}^{\prime \prime} \alpha c_{k}\right) \cdot c_{i} \\
& =\sum_{\left(c_{i}\right)\left(c_{j}\right)} c_{j}^{\prime} c_{i}^{\prime} \otimes\left(c_{j}^{\prime \prime} \alpha c_{k}\right) \cdot c_{i}^{\prime \prime} \\
& =\sum_{\left(c_{i}\right)\left(c_{j}\right)} c_{j}^{\prime} c_{i}^{\prime} \otimes c_{j}^{\prime \prime} c_{i}^{\prime \prime}(\alpha \cdot i) c_{k} c_{i}^{\prime \prime \prime},
\end{aligned}
$$

then $\delta_{L}\left(\left(c_{j} \alpha c_{k}\right) \cdot c_{i}\right)=\delta_{L}\left(c_{j} \alpha c_{k}\right) \cdot c_{i}$.

$\delta_{R}$ can be discussed similarly.

Therefore, $k\left(Q_{1}, \mathcal{C}\right)$ is a $k\left(Q_{0}, \mathcal{C}\right)$-Hopf bimodule.

The $\mathcal{C}$-arrow-stability of $k\left(Q^{c}, \mathcal{C}\right)$ under the left and right module actions of the $k\left(Q_{0}, \mathcal{C}\right)$-Hopf bimodule strcutre of $k\left(Q_{1}, \mathcal{C}\right)$ follows from (1) and (2) and Definition 2.1. We prove this only for the left module action as follows.

For six non-zero elements $c_{i}, d_{i} \in C_{i}, c_{j}, d_{j} \in C_{j}, c_{k}, d_{k} \in C_{k}$, we have $\widetilde{P}\left(c_{i}\right)=\widetilde{P}\left(d_{i}\right)=v_{i}, \widetilde{P}\left(c_{j} \alpha c_{k}\right)=\widetilde{P}\left(d_{j} \alpha d_{k}\right)=\alpha$, and

$$
\begin{aligned}
\widetilde{P}\left(c_{i} \cdot\left(c_{j} \alpha c_{k}\right)\right) & =\widetilde{P}\left(\sum_{\left(c_{i}\right)}\left(c_{i}^{\prime} c_{j}\right)(i \cdot \alpha)\left(c_{i}^{\prime \prime} c_{k}\right)\right)=\widetilde{P}(i \cdot \alpha) \\
& =\widetilde{P}\left(\sum_{\left(d_{i}\right)}\left(d_{i}^{\prime} d_{j}\right)(i \cdot \alpha)\left(d_{i}^{\prime \prime} d_{k}\right)\right) \\
& =\widetilde{P}\left(d_{i} \cdot\left(d_{j} \alpha d_{k}\right)\right)
\end{aligned}
$$

Note that by the condition of $G$-type, i.e. Definition 2.3 (ii), $\sum_{\left(c_{i}\right)}\left(c_{i}^{\prime} c_{j}\right)(i$. $\left.\alpha)\left(c_{i}^{\prime \prime} c_{k}\right)\right) \neq 0$ and $\sum_{\left(d_{i}\right)}\left(d_{i}^{\prime} d_{j}\right)(i \cdot \alpha)\left(d_{i}^{\prime \prime} d_{k}\right) \neq 0$. Thus, the $\mathcal{C}$-arrow-stability of $k\left(Q^{c}, \mathcal{C}\right)$ follows under the left module action. 
(ii) $\Rightarrow$ (iii): The main part of this proof was given in [6] except for the $\mathcal{C}$-arrowstability.

Denote by $\delta_{L}$ and $\delta_{R}$ respectively the left coaction and right coaction of the $k\left(Q_{0}, \mathcal{C}\right)$-Hopf bimodule $k\left(Q_{1}, \mathcal{C}\right)$. Define $\psi_{0}: X=k\left(Q^{c}, \mathcal{C}\right) \otimes k\left(Q^{c}, \mathcal{C}\right) \rightarrow k\left(Q_{0}, \mathcal{C}\right)$ to be the composition of

$$
X \stackrel{p_{0} \otimes p_{0}}{\longrightarrow} k\left(Q_{0}, \mathcal{C}\right) \otimes k\left(Q_{0}, \mathcal{C}\right) \stackrel{m_{0}}{\longrightarrow} k\left(Q_{0}, \mathcal{C}\right)
$$

where $m_{0}$ means the multiplication in $k\left(Q_{0}, \mathcal{C}\right), p_{0}$ is the projection from $k\left(Q^{c}, \mathcal{C}\right)$ to $k\left(Q_{0}, \mathcal{C}\right)$.

(1) $\psi_{0}$ is a coalgebra map.

Use the subindex to denote the length of a generalized path. For example, if we denote a generalized path by $x_{i}$, then the length of $x_{i}$ is $i$. Thus, in general, we can write an element in $X$ by $\sum_{s, t, i, j} \alpha_{i j}^{s t}\left(x_{i}^{s} \otimes x_{j}^{t}\right)$ for $\alpha_{i j}^{s t} \in k$. Therefore,

$$
\Delta \psi_{0}\left(\sum_{s, t, i, j} \alpha_{i j}^{s t}\left(x_{i}^{s} \otimes x_{j}^{t}\right)\right)=\Delta\left(\sum_{s, t} \alpha_{00}^{s t} x_{0}^{s} x_{0}^{t}\right)=\sum_{s, t} \alpha_{00}^{s t} \Delta\left(x_{0}^{s}\right) \Delta\left(x_{0}^{t}\right)
$$

and

$$
\begin{aligned}
\left(\psi_{0} \otimes \psi_{0}\right) \Delta\left(\sum_{s, t, i, j} \alpha_{i j}^{s t}\left(x_{i}^{s} \otimes x_{j}^{t}\right)\right) & =\left(\psi_{0} \otimes \psi_{0}\right)(i d \otimes \tau \otimes i d)\left(\sum_{s, t, i, j} \alpha_{i j}^{s t} \Delta\left(x_{i}^{s}\right) \otimes \Delta\left(x_{j}^{t}\right)\right) \\
& =\left(\psi_{0} \otimes \psi_{0}\right)\left(\sum_{s, t, i, j} \alpha_{i j}^{s t}\left(x_{i}^{s}\right)^{\prime} \otimes\left(x_{j}^{t}\right)^{\prime} \otimes\left(x_{i}^{s}\right)^{\prime \prime} \otimes\left(x_{j}^{t}\right)^{\prime \prime}\right) \\
& =\sum_{s, t, i, j} \alpha_{i j}^{s t}\left(x_{i}^{s}\right)^{\prime} \otimes\left(x_{j}^{t}\right)_{0}^{\prime} \otimes\left(x_{i}^{s}\right)^{\prime \prime} 0\left(x_{j}^{t}\right)^{\prime \prime} 0 \\
& =\sum_{s, t} \alpha_{00}^{s t}\left(x_{0}^{s}\right)^{\prime}\left(x_{0}^{t}\right)^{\prime} \otimes\left(x_{0}^{s}\right)^{\prime \prime}\left(x_{0}^{t}\right)^{\prime \prime} \\
& =\sum_{s, t} \alpha_{00}^{s t} \Delta\left(x_{0}^{s}\right) \Delta\left(x_{0}^{t}\right)
\end{aligned}
$$

then $\Delta \psi_{0}=\left(\psi_{0} \otimes \psi_{0}\right) \Delta$. Note that $\Delta$ on the right-side of the above equalities is the comultiplication of $X$. Clearly, $\varepsilon \psi_{0}=\varepsilon$. Thus $\psi_{0}$ is a coalgebra map.

Define $\psi_{1}: X=k\left(Q^{c}, \mathcal{C}\right) \otimes k\left(Q^{c}, \mathcal{C}\right) \longrightarrow k\left(Q_{1}, \mathcal{C}\right)$ to be the composition of

$$
X \stackrel{p_{0} \otimes p_{1}+p_{1} \otimes p_{0}}{\longrightarrow}\left(k\left(Q_{0}, \mathcal{C}\right) \otimes k\left(Q_{1}, \mathcal{C}\right)\right)+\left(k\left(Q_{1}, \mathcal{C}\right) \otimes k\left(Q_{0}, \mathcal{C}\right)\right) \stackrel{m_{l}+m_{r}}{\longrightarrow} k\left(Q_{1}, \mathcal{C}\right)
$$

where $m_{l}$ and $m_{r}$ denote the left and right module actions respectively. 
(2) $\psi_{1}$ is a $k\left(Q_{0}, \mathcal{C}\right)$-bicomodule map.

In fact, by Proposition $2.1, k\left(Q_{n}, \mathcal{C}\right)$ is a $k\left(Q_{0}, \mathcal{C}\right)$-bicomodule via

$$
\delta_{L}(x):=\sum_{(x)}\left(x^{\prime}\right)_{0} \otimes x^{\prime \prime} \quad \text { and } \quad \delta_{R}(x):=\sum_{(x)} x^{\prime} \otimes\left(x^{\prime \prime}\right)_{0}
$$

where $x \in k\left(Q_{n}, \mathcal{C}\right), n \geq 0$. Let $\sum_{s, t, i, j} \alpha_{i j}^{s t}\left(x_{i}^{s} \otimes x_{j}^{t}\right)$ be an element in $X$. Then

$$
\psi_{1}\left(\sum_{s, t, i, j} \alpha_{i j}^{s t}\left(x_{i}^{s} \otimes x_{j}^{t}\right)\right)=\sum_{s, t} \alpha_{01}^{s t} x_{0}^{s} \cdot x_{1}^{t}+\sum_{s, t} \alpha_{10}^{s t} x_{1}^{s} \cdot x_{0}^{t}
$$

therefore

$$
\delta_{L} \psi_{1}\left(\sum_{s, t, i, j} \alpha_{i j}^{s t}\left(x_{i}^{s} \otimes x_{j}^{t}\right)\right)=\sum_{s, t} \alpha_{01}^{s t} x_{0}^{s} \cdot \delta_{L}\left(x_{1}^{t}\right)+\sum_{s, t} \alpha_{10}^{s t} \delta_{L}\left(x_{1}^{s}\right) \cdot x_{0}^{t}
$$

On the other hand,

$$
\begin{aligned}
\left(i d \otimes \psi_{1}\right) \delta_{L}\left(\sum_{s, t, i, j} \alpha_{i j}^{s t}\left(x_{i}^{s} \otimes x_{j}^{t}\right)\right) & =\left(i d \otimes \psi_{1}\right)\left(\sum_{s, t, i, j} \alpha_{i j}^{s t} \psi_{0}\left(\left(x_{i}^{s}\right)^{\prime} \otimes\left(x_{j}^{t}\right)^{\prime}\right) \otimes\left(\left(x_{i}^{s}\right)^{\prime \prime} \otimes\left(x_{j}^{t}\right)^{\prime \prime}\right)\right) \\
& =\left(i d \otimes \psi_{1}\right)\left(\sum_{s, t, i, j} \alpha_{i j}^{s t}\left(\left(x_{i}^{s}\right)^{\prime}\right)_{0}\left(\left(x_{j}^{t}\right)^{\prime}\right)_{0} \otimes\left(\left(x_{i}^{s}\right)^{\prime \prime} \otimes\left(x_{j}^{t}\right)^{\prime \prime}\right)\right) \\
& =\sum_{s, t, i, j} \alpha_{i j}^{s t}\left(\left(x_{i}^{s}\right)^{\prime}\right)_{0}\left(\left(x_{j}^{t}\right)^{\prime}\right)_{0} \otimes\left(\left(x_{i}^{s}\right)^{\prime \prime}\right)_{0} \cdot\left(\left(x_{j}^{t}\right)^{\prime \prime}\right)_{1} \\
& +\sum_{s, t, i, j} \alpha_{i j}^{s t}\left(\left(x_{i}^{s}\right)^{\prime}\right)_{0}\left(\left(x_{j}^{t}\right)^{\prime}\right)_{0} \otimes\left(\left(x_{i}^{s}\right)^{\prime \prime}\right)_{1} \cdot\left(\left(x_{j}^{t}\right)^{\prime \prime}\right)_{0} \\
& =\sum_{s, t, i, j} \alpha_{i j}^{s t}\left(x_{i}^{s}\right)_{0} \delta_{L}\left(\left(x_{j}^{t}\right)_{1}\right)+\sum_{s, t, i, j} \alpha_{i j}^{s t} \delta_{L}\left(\left(x_{i}^{s}\right)_{1}\right)\left(x_{j}^{t}\right)_{0} \\
& =\sum_{s, t} \alpha_{01}^{s t} x_{0}^{s} \cdot \delta_{L}\left(x_{1}^{t}\right)+\sum_{s, t} \alpha_{10}^{s t} \delta_{L}\left(x_{1}^{s}\right) \cdot x_{0}^{t} .
\end{aligned}
$$

Thus, $\delta_{L} \psi_{1}=\left(i d \otimes \psi_{1}\right) \delta_{L}$ and $\psi_{1}$ is a left $k\left(Q_{0}, \mathcal{C}\right)$-comodule map. Note that $\delta_{L}$ on the right-side of the above equalities is the left $k\left(Q_{0}, \mathcal{C}\right)$-comodule structure map of $X$. Similarly we can prove that $\delta_{R} \psi_{1}=\left(\psi_{1} \otimes i d\right) \delta_{R}$ and $\psi_{1}$ is a right $k\left(Q_{0}, \mathcal{C}\right)$-comodule map. Hence $\psi_{1}$ is a $k\left(Q_{0}, \mathcal{C}\right)$-bicomodule map.

Moreover, define $\psi_{n}: X \stackrel{\triangle^{(n-1)}}{\rightarrow} X \otimes X \otimes \cdots \otimes X \stackrel{\psi_{1}^{\otimes n}}{\rightarrow} k\left(Q_{1}, \mathcal{C}\right)^{\otimes n}$ for $n \geq 2$ and define $\psi=\sum \psi_{n}$. Then $\psi: k\left(Q^{c}, \mathcal{C}\right) \otimes k\left(Q^{c}, \mathcal{C}\right)=X \rightarrow \operatorname{CoT}_{k\left(Q_{0}, \mathcal{C}\right)}\left(k\left(Q_{1}, \mathcal{C}\right)\right) \cong$ $k\left(Q^{c}, \mathcal{C}\right)$ is associative since $\Delta$ is coassociative and $\otimes$ is associative where the 


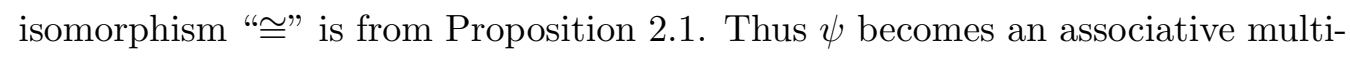
plication in $k\left(Q^{c}, \mathcal{C}\right)$.

By (1), (2) and Proposition 3.9,

$$
\psi: k\left(Q^{c}, \mathcal{C}\right) \otimes k\left(Q^{c}, \mathcal{C}\right) \rightarrow k\left(Q^{c}, \mathcal{C}\right)
$$

is a coalgebra map. This means that $k\left(Q^{c}, \mathcal{C}\right)$ is a bialgebra. Due to the known conditions, the coradical $k\left(Q_{0}, \mathcal{C}\right)$ of $k\left(Q^{c}, \mathcal{C}\right)$ is a Hopf algebra meanwhile it is a sub-bialgebra of $k\left(Q^{c}, \mathcal{C}\right)$ as bialgebra. Therefore, by Proposition $3.8, k\left(Q^{c}, \mathcal{C}\right)$ is a Hopf algebra.

It is graded with length grading from the definition of the multiplication $\psi$.

Due to the $\mathcal{C}$-arrow-stability under the left and right module actions in Hopf bimodule structure, $k\left(Q^{c}, \mathcal{C}\right)$ is $\mathcal{C}$-arrow-stable under $\psi_{1}$, then moreover under $\psi$. That is, $k\left(Q^{c}, \mathcal{C}\right)$ is $\mathcal{C}$-arrow-stable under the multiplication of this Hopf algebra structure.

(iii) $\Rightarrow($ i): By Proposition 3.6.

Acknowledgement The authors take this opportunity to express thanks to the referee for the helpful and important suggestions.

\section{References}

[1] C.Cibils and M.Rosso, Hopf quivers, J. Algebra, 254: 241-251 (2002).

[2] F.U.Coelho and S.X.Liu, Generalized path algebras, In: Interactions between ring theory and repersentations of algebras (Murcia), Lecture Notes in Pure and Appl. Math, Marcel-Dekker, New York, 210, 2000, pp.53-66.

[3] I.Kaplansky, Bialgebras, University of Chicago, Chicago, 1975.

[4] F.Li, Characterization of left Artinian algebras through pseudo path algebras, J. Australia Math. Soc., 83: 385-416 (2007).

[5] F.Li and G.X.Liu, Generalized path coalgebra and its application to dual Gabriel Theorem, Acta Math. Sinica (Chinese Series), 51(5): 853-862 (2008). 
[6] G.X.Liu, Classification of finite dimensional basic Hopf algebras and related topics, Doctoral Dissertation, Zhejiang University, China (2005).

[7] S.Montgomery, Hopf Algebras and Their Actions on Rings, CBMS, Lecture in Math, Providence, RI, 82, 1993.

[8] W.D.Nichols, Bialgebras of type one, Comm. in Algebra, 6: 1521-1552 (1978).

[9] F. van Oystaeyen and P.Zhang, Quivers and Hopf Algebras, In: Nonlin. evolution equations and Dynamical sys., Proc. ICM 2002 Satellite Conference.

[10] M.Takeuchi, Free Hopf algebras generated by coalgebras, Journal of Math. Soc. Japan, 23(4): 560-581 (1971).

Fang Li

Department of Mathematics

Zhejiang University

Hangzhou, Zhejiang 310027

China

Email: fangli@cms.zju.edu.cn

Lili Chen

Department of Mathematics

Qingdao University of Science and Technology (Laoshan Campus)

Qingdao, Shandong 266061

China

Email: lilychen0229@yahoo.com.cn 